\title{
Quality Improvement for Exemplar-based Image Inpainting using a Modified Searching Mechanism
}

\author{
Mariwan Wahid Ahmed 1 , Alan Anwer Abdulla ${ }^{2}$ \\ ${ }^{1}$ Department of Computer, College of Science, University of Sulaimani, Sulaymaniyah, Iraq, ${ }^{2}$ Department of Information \\ Technology, College of Commerce, University of Sulaimani, Sulaymaniyah, Iraq, ${ }^{2}$ Department of Information Technology, \\ University College of Goizha, Sulaymaniyah, Iraq
}

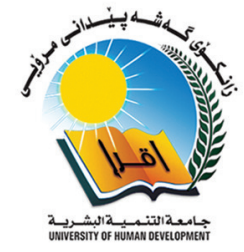

\section{A B S T R A C T}

Digital image processing has a significant impact in different research areas including medical image processing, biometrics, image inpainting, object detection, information hiding, and image compression. Image inpainting is a science of reconstructing damaged parts of digital images and filling-in regions in which information are missing which has many potential applications such as repairing scratched images, removing unwanted objects, filling missing area, and repairing old images. In this paper, an image inpainting algorithm is developed based on exemplar, which is one of the most important and popular images inpainting technique, to fill-in missing area that caused either by removing unwanted objects, by image compression, by scratching image, or by image transformation through internet. In general, image inpainting consists of two main steps: The first one is the priority function. In this step, the algorithm decides to select which patch has the highest priority to be filled at the first. The second step is the searching mechanism to find the most similar patch to the selected highest priority patch to be inpainted. This paper concerns the second step and an improved searching mechanism is proposed to select the most similar patch. The proposed approach entails three steps: (1) Euclidean distance is used to find the similarity between the highest priority patches which need to be inpainted with each patch of the input image, (2) the position/location distance between those two patches is calculated, and (3) the resulted value from the first step is summed with the resulted value obtained from the second step. These steps are repeated until the last patch from the input image is checked. Finally, the smallest distance value obtained in step 3 is selected as the most similar patch. Experimental results demonstrated that the proposed approach gained a higher quality in terms of both objectives and subjective compared to other existing algorithms.

Index Terms: Image Inpainting, Sum of Square Difference, Image Quality, Peak Signal-to-noise Ratio, Position Distance

\section{INTRODUCTION}

The image processing techniques have been developed in the various of areas such as remote sensing, video processing, image inpainting, medical image processing, pattern

\section{Access this article online}

DOI: 10.21928/uhdjst.v4n1y2020.pp1-8

E-ISSN: $2521-4217$

P-ISSN: 2521-4209

Copyright (C) 2020 Ahmed and Abdulla. This is an open access article distributed under the Creative Commons Attribution Non-Commercial No Derivatives License 4.0 (CC BY-NC-ND 4.0) recognition, biometrics, traffic systems, image compression, information hiding [1], and printing industry [2]. Image inpainting is the process of reconstructing the damaged image and/or removing an object in an image [3], [4]. The fundamental concept of image inpainting is to fill the missing regions of an image using the surrounding information of that region. Image inpainting techniques entail many applications such as automatic text removal and/or object removal in images/films for special effects [5], deleting of blurs of dust in image, red-eye correction, inventive effect by deleting object [6], [7], remove video logos [8], old image/film restoration [9], and medical image correction

Corresponding author's e-mail: Alan Anwer Abdulla, Department of Information Technology, College of Commerce, University of Sulaimani and University college of Goizha, Sulaimani, Iraq. E-mail: Alan.abdulla@univsul.edu.iq

Received: 24-12-2019

Accepted: 19-01-2020

Published: 21-01-2020 
(image inpainting use to complete missing or distorted information in medical images) [10]. In general, image inpainting techniques are categorized into five major types which are: (1) Partial differential equation based inpainting, (2) texture synthesis based inpainting, (3) exemplar-based inpainting, (4) semi-automatic and fast inpainting, and (5) hybrid inpainting [11], [12]. Each of these types has its own advantages and limitations and each of them recovers the damaged regions in accordance with certain requirements of expectation of the repaired image content. This paper focuses on the third type, i.e., exemplar-based image inpainting.

The exemplar-based image inpainting is one of the most important types of inpainting techniques and it has been proved to be the most effective type of inpainting [13]. Exemplar-based image inpainting mainly includes two main steps. The first step is the priority assignment, in this step, one patch from the boundary of the missing region with the highest priority must be selected using one of the priority functions and it must be filled at first. The second step involves searching for the best-matched patch based on one of the searching mechanisms. In this area of research, researchers try to improve one or both of these steps to obtain better results in terms of the quality of the reconstructed image. This paper focuses on improving the searching mechanism by taking advantage from the distance between the patch to be inpainted and the other best match patches.

The rest of the paper is organized as follows: In section 2, the literature review is presented. Section 3 illustrates the statement of the problem. Section 4 presents the proposed inpainting approach. The experimental results are shown in Section 5. Finally, our conclusions are given in Section 6.

\section{LITERATURE REVIEW}

Different categories of image inpainting are mentioned in the previous section. Since the contribution in this paper concerns on the exemplar-based image inpainting, therefore this section is going to review some important approaches related to exemplar-based image inpainting.

The idea of exemplar-based inpainting is first invented by Perez et al. in 2003 [14]. The core of this algorithm is an isophote driven image sampling process, isophote refers to the direction and intensity of the patch center point. Patch can be defined as a small (generally rectangular) piece/block of an image. Essentially, in image inpainting, $\Psi$ p refers to the targeted patch in which some of its pixels values are missing and $\Psi \mathrm{q}$ refers to the best match founded patch. Moreover, one of the most important steps in image inpainting is from which patch (out of all the patches located around the border of the missing area) should the system start to inpainting; this process refers to priority function and it denotes as $\mathrm{P}(\mathrm{p})$. In Perez et al. [14], the priority function of their algorithm is defined by a production of confidence term $\mathrm{C}(\mathrm{p})$ and data term $\mathrm{D}(\mathrm{p})$.

$$
\mathrm{P}(\mathrm{p})=\mathrm{C}(\mathrm{p}) \times \mathrm{D}(\mathrm{p})
$$

Moreover, the confidence term represents texture information and data term represents structure information. These two parameters decide which patch has the highest priority. Furthermore, $\Psi_{\mathrm{p}}$ will be filled by the $\Psi \mathrm{q}$, only the missing part of the $\Psi$ p will replace by the $\Psi$ q. In each iteration, once the best match patch $\Psi_{\mathrm{q}}$ is founded, it will immediately use to fill the missing part of the targeted patch $\Psi$ p and then the next iteration will start to find the best match patch $\Psi$ q for the next targeted patch $\Psi$ p. This process continues until all the targeted patches are filled completely [14].

The extended version of the previous work was published by Perez et al. in 2004 with a more detailed description of the algorithm and extensive experiments [15].

In general, each exemplar-based image inpainting approach includes two main steps. The first one is the priority function that uses to decide from which patch, out of all the patches located around the boundary of the missing area, the algorithm should start. On the other hand, the second phase is the searching mechanism using distance technique, for example, sum of square difference that is used to select the best-matched patch based on the difference between the targeted patch $\Psi \mathrm{p}$ and the best match patch $\Psi \mathrm{q}$. Therefore, all the approaches proposed in this research area are either improve the priority function, improve the way of finding differences between $\Psi_{\mathrm{p}}$ and $\Psi_{\mathrm{q}}$, or improve both steps. In addition, the essential purpose of the competition in this research area is to improve the quality of the reconstructed image.

Wen-Huang et al. highlighted the limitation of the previous algorithm in Perez et al. [14] and Perez et al. [15] in which the defined priority function quickly drops the value of the confidence term $\mathrm{C}(\mathrm{p})$ to zero [16]. Consequently, this leads to create a problem in which the priority function $\mathrm{P}(\mathrm{p})$ is gradually depend on only structure information $\mathrm{D}(\mathrm{p})$ without making the texture information $\mathrm{C}(\mathrm{p})$ into consideration; 
therefore, the priority function is not able to find the highest priority patch correctly. This kind of drawback is known as the dropping effect. To overcome this drawback, Wen-Huang et. al. proposed an algorithm in which defined a regularized function $\mathrm{Rc}(\mathrm{p})$ instead of confidence term $\mathrm{C}(\mathrm{p})$ as follows:

$$
\mathrm{R}_{\mathrm{c}}(\mathrm{p})=(1-\omega) \times \mathrm{C}(\mathrm{p})+\omega
$$

Where $\omega$ is the regularizing factor for controlling curve smoothness and takes a value between 0 and 1 . In general, in this work, $\omega$ sets to 0.7 . Finally, the equation changed as:

$$
R P(p)=\alpha \cdot R_{c}(p)+\beta \cdot D(p)
$$

Where $0 \leq \alpha, \beta \leq 1$, and $\alpha+\beta=1$. Authors claimed that their proposed approach is improved the quality of the reconstructed image [16].

In the previously reviewed approaches, only one patch $\Psi \mathrm{q}$ is used to reconstruct the inpainted patch $\Psi \mathrm{p}$. In 2008, Alexander et al. proposed a new mechanism to fill the inpainted patches using multiple samples within the image and weight their contribution according to their similarity to the neighborhood under evaluation. Using the weighted aggregation of multiple patches yields a much-improved result [17].

In 2015, Huang et al. defined a new priority function for exemplar-based image inpainting [18]. Different from the priority function defined in Perez et al. [14], Hsieh et al. [16], this proposed priority function first rely on the structure information $\mathrm{D}(\mathrm{p})$, and then used texture information $\mathrm{C}(\mathrm{p})$. In other words, this priority function works on the $\mathrm{D}(\mathrm{p})$ and $\mathrm{C}(\mathrm{p})$ separately. This approach works well for the case of the curve or cross-shaped structures. The presented results in this paper show that this approach is able to recover image geometry and texture well compared to other existing approaches [18].

A new mechanism was proposed by Liu et al., in 2018, in which the proposed algorithm added boundary-constrained similar patches to find more suitable similar patches and boundary filling instead of patch filling. To measure the similarity between boundaries, they used boundary matching distance. This type of distance measurement was applied for the boundary matching algorithm. This proposed algorithm can repair structural and texture complex images very well [19].

Awati et al., in 2018, proposed a new technique to enhance the quality of exemplar-based image inpainting [20]. This algorithm upgrades the image inpainting approaches mainly for these images that involve edges and corner information. This approach uses fractional derivative and additional curvature finding methods for finding data term in patch priority.

The proposed approach presents in this paper will modify the searching mechanism, while uses the same priority function presented in Hsieh et al. [16], more details will be given in the next section.

\section{STATEMENT OF THE PROBLEM}

The digital image may cause to lose some of it is regions affecting by removing unwanted objects, by image compression, by scratching image, or by image transformation through internet. Image inpainting is becoming a common tool for repairing the scratched or damaged area of an image. It entails reconstructing damaged parts and filling-in regions in which data/color information are missing.

\section{PROPOSED APPROACH}

This section gives the steps of the proposed approach in detail. It is assumed that the source region, target region, and the boundary denote by $\Phi, \Omega$, and $\delta \Omega$, respectively. Furthermore, the target patch denotes by $\Psi_{\mathrm{p}}$ in which its center point is $\mathrm{p} \delta \Omega$ [21]. The proposed approach consists of the following steps:

\subsection{Manually Select the Target Region $\Omega$}

Selecting the target region means to select the degraded region or unwanted region to be filled using the surrounding information.

In this step, the target region is selected by marking this area using a specific color, for example, "black color," and then the mask image is also created. Mask image is a binary image in which zero pixels indicate the target region and the ones are showing the remaining part of the image, as illustrated in Fig. 1.

\subsection{Target Region's Boundary Detection $\delta \Omega$}

One of the most important steps of developing the inpainting algorithm is the boundary detection of the target region because the selection of the highest priority patch from the boundary of its target region can be decided. The boundary of the target region should be detected using one of the edge detection techniques such as Prewitt, Sobel, Canny, or Laplacian edge detection see Fig. 2. 


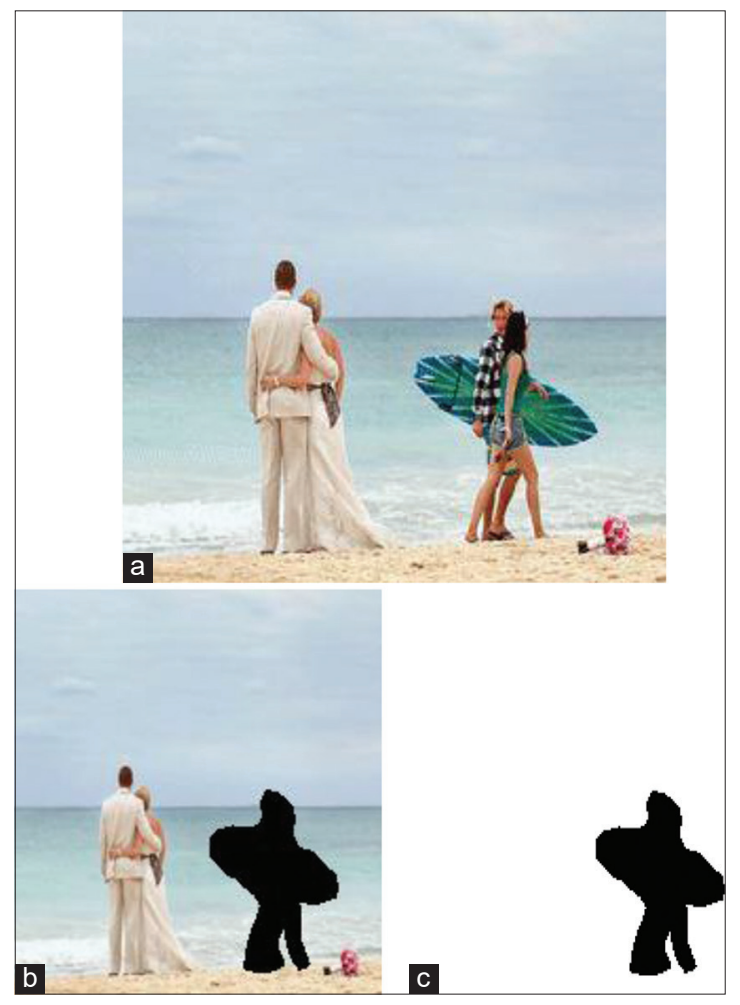

Fig. 1. (a) Original image, (b) mage with target region, (c) mask image.

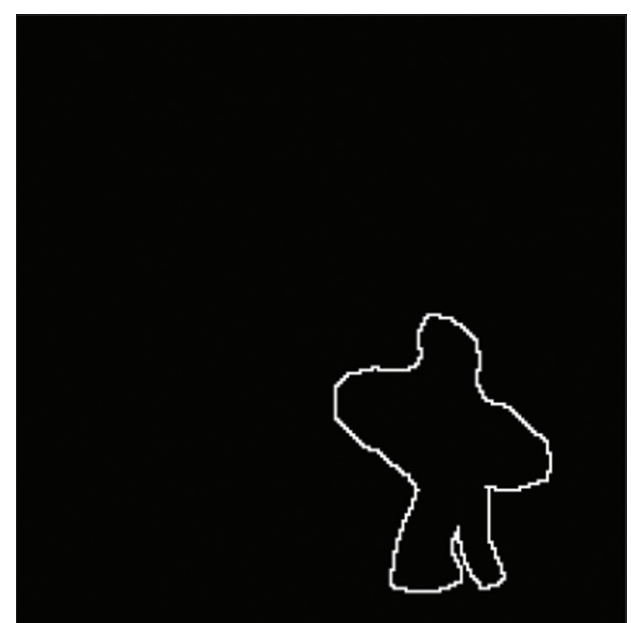

Fig. 2. Boundary Detection of the Target Region using Laplacian edge detection.

\subsection{Patch Priority Function}

It is useful to decide which patch on the boundary of the target region must be inpainted first. After patch priority function is computed, the patch $\Psi \mathrm{p}$ centered at the point $\mathrm{p}$ for all p \& $\delta \Omega$ with the highest priority is taken, see Fig. 3 . In this proposed approach, the same patch priority equation presented in Hsieh et al. [16] is performed.

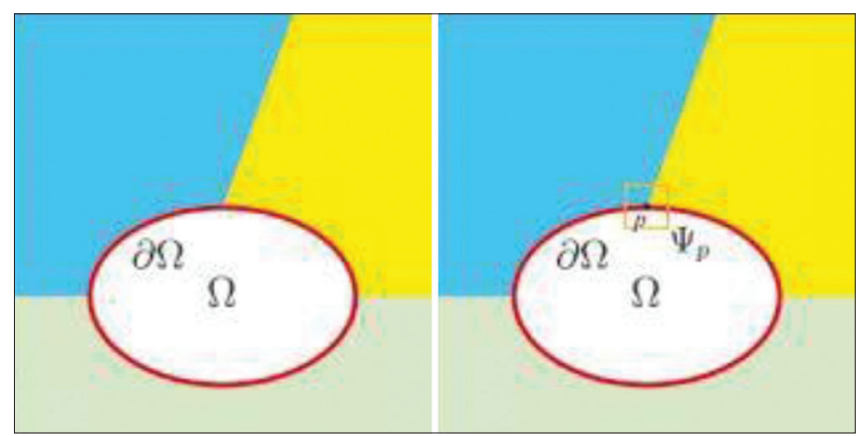

Fig. 3. Select $\Psi p$ from $\delta \Omega$ with the highest priority.

\subsection{Searching for the Most Similar Patch}

Once the highest priority patch $\Psi \mathrm{p}$ is selected on the boundary of the target region $\delta \Omega$ in the previous step, the most similar patch to fill the unknown pixels of the $\Psi$ p needs to be found. For this sake, the proposed approach developed an improved searching mechanism in which the entire source region $\Phi$ of the image should be searched patch by patch from the upper left corner of the image. The source region $\Phi$ consists of the whole image without the target region (I- $\Omega$ ). Now for each patch from the source region that is denoted by $\Psi$ q, two different distances between $\Psi \mathrm{p}$ and $\Psi \mathrm{q}_{\mathrm{i}}$ need to be found. For the first one, the distance between the positions of each patch on the image is calculated.

$$
\begin{gathered}
\text { X_POS }=\left|\Psi \mathrm{p}_{\mathrm{x}}-\Psi \mathrm{q}_{\mathrm{xi}}\right| \\
\mathrm{Y} \_\mathrm{POS}=\left|\Psi_{\mathrm{p}}-\Psi \mathrm{q}_{\mathrm{yi}}\right| \\
\text { DISTANCE_POS }=\frac{\mathrm{X} \_\mathrm{POS}+\mathrm{Y} \_P O S}{\mathrm{img} \operatorname{size} / \lambda}
\end{gathered}
$$

$K$ is a positive number of uses to make a balance between the position's distance and Euclidean distance. In this proposed approach $K$ is set to 10, and X_POS calculates a position distance between $\Psi \mathrm{p}$ and $\Psi \mathrm{q}$ on the $\mathrm{X}$-axis and Y_POS for Y-axis.

For the second one, the Euclidean distance between these two patches is calculated as follows:

$$
\begin{gathered}
\hat{\mathrm{R}}=\left(\mathrm{R}_{\Psi_{\mathrm{p}}}-\mathrm{R}_{\Psi_{\mathrm{q}}}\right)^{2} \\
\hat{\mathrm{G}}=\left(\mathrm{G}_{\Psi_{\mathrm{p}}}-\mathrm{G}_{\Psi_{\mathrm{q}} \mathrm{i}}\right)^{2} \\
\hat{\mathrm{B}}=\left(\mathrm{B}_{\Psi_{\mathrm{p}}}-\mathrm{B}_{\Psi \mathrm{qi}}\right)^{2} \\
\mathrm{ED}=\sqrt{(\hat{\mathrm{R}}+\hat{\mathrm{G}}+\hat{\mathrm{B}}) / 3}
\end{gathered}
$$

Where, R, G, and B represent the red, green, and blue channels of the image, respectively. 


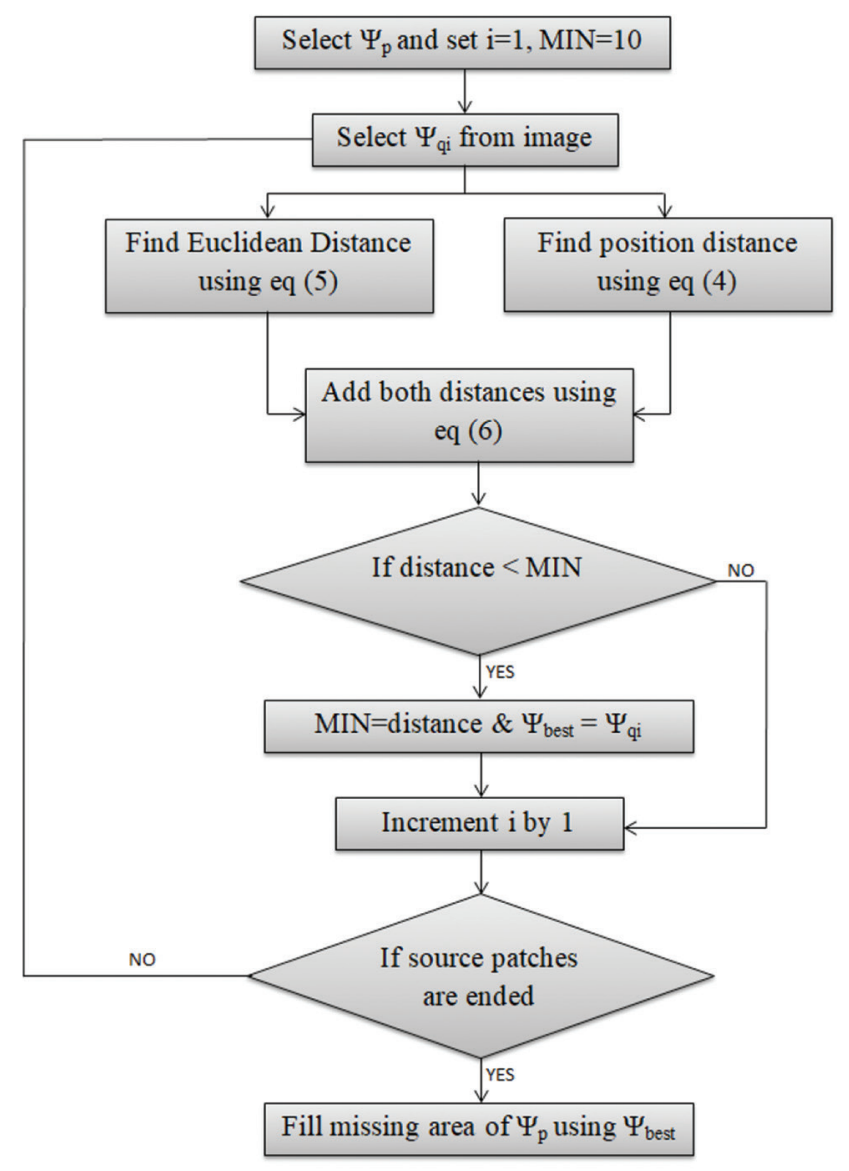

Fig. 4. Block diagram of the proposed searching mechanism.

Finally, we perform the summation between these two distances value to obtain the appropriate distance value.

$$
\text { Distance=DISTANCE_POS+ED }
$$

The above equations should repeat between $\Psi \mathrm{p}$ and the remaining patches in the source region $\Psi \mathrm{q}_{\mathrm{i}}$. In the end, the minimum distance value is chosen as the best similar patch. This process continues until the entire target region is filled.

The following diagram illustrates the proposed searching mechanism.

Fig. 4 is illustrated our proposed searching mechanism to fill the missing pixels in the highest priority patch $\Psi \mathrm{p}$ that is selected in section 4.3. Consequently, the algorithm searches the entire image patch by patch. These patches are denoted by $\Psi \mathrm{q}_{\mathrm{i}}$ where $i$ is a number of patches in the image that starts from one to the last patch number. After performing

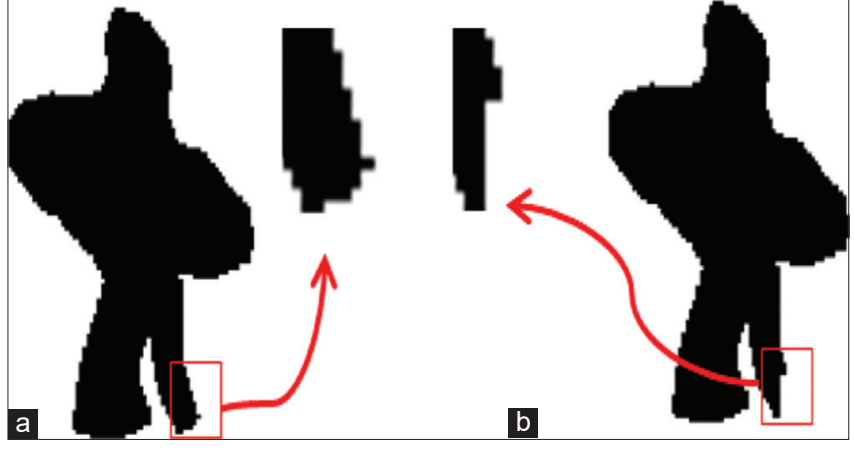

Fig. 5. (a) Mask image before the confidence value is updated, (b) mask image after the confidence value is updated at the first iteration.

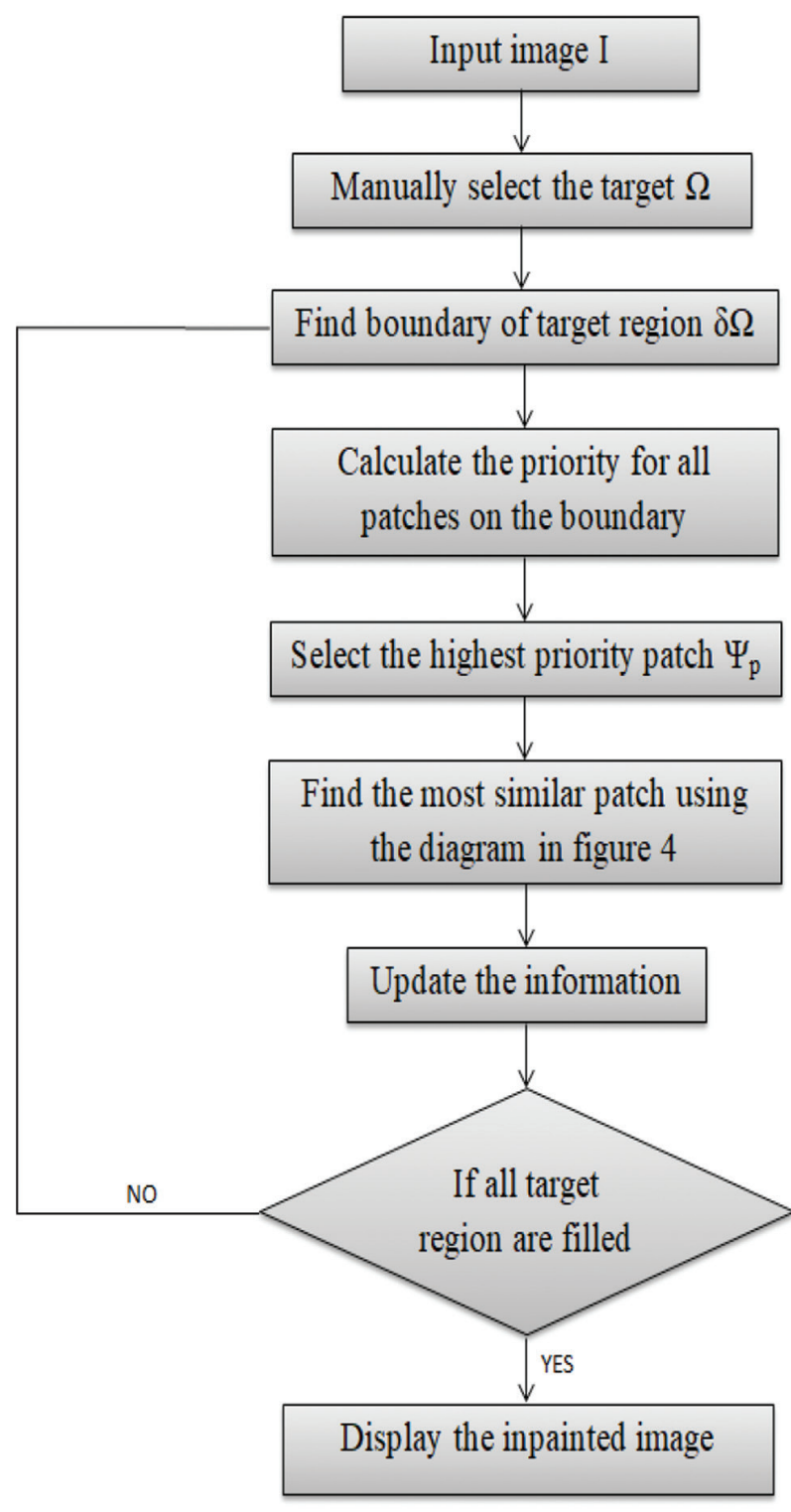

Fig. 6. Block diagram of the proposed approach. 
equations of 4, 5, and 6 , the result of equation 6 needs to be checked whether it is smaller than the MIN value or not (MIN is a variable used to take the smaller distance value in each iteration that is initially set to 10) if it is smaller than the MIN variable, it means that the current patch $\left(\Psi_{q_{i}}\right)$ is the most similar to $\Psi \mathrm{p}$ than the previous patch $\left(\Psi_{\mathrm{q}_{\mathrm{i}-1}}\right)$ ? This process continues until the last $i$. Finally, we set $\Psi_{\text {best }}$ to the most similar patch and replace the missing pixels of $\Psi$ p using $\Psi_{\text {best }}$

\subsection{Updating Confidence Value}

After the patch $\Psi_{\mathrm{p}}$ has been filled as explained in the previous step, the region of $\Psi \mathrm{p}$ in the confidence $C(\mathrm{p})$ on the mask image is updated as follow:

$C(p)=C\left(p^{\prime}\right)$ where $C\left(p^{\prime}\right)$ is the region of the $\Psi p$ after it is filled.

Fig. 5 shows updating confidence value at the first iteration.

Fig. 6 shows the block diagram of the whole steps of the proposed approach.

\section{RESULTS AND COMPARISON}

To evaluate the performance of the proposed approach, experiments are conducted extensively using different natural and complex images of different file formats such as. JPG and.PNG. These images are common images that are usually used in image inpainting research area and they are from a dataset [22]. Fig. 7 shows some images which are used in the experiments: The second column includes original images and the third column contains images which marked the target region in black color.

The proposed approach is compared with algorithms in Perez et al. [14] and Huang et al. [18]. Two main kinds of quality assessment techniques are performed (objective and subjective). Fig. 8 illustrates the results of reconstructing the target region of the images in Fig. 7 using each of the proposed approaches as well as approaches in Perez et al. [14] and Huang et al. [18]. The reconstructed of the missing region is highlighted in a red circle.

In Fig. 8, some of them resulted images cannot be seen subjectively, i.e., visually. The peak signal-to-noise ratio (PSNR) is used as a quality assessment objectively to measure the quality of the reconstructed images. Results demonstrate that the proposed approach obtained the highest PSNR value compared to other tested approaches, Table 1.

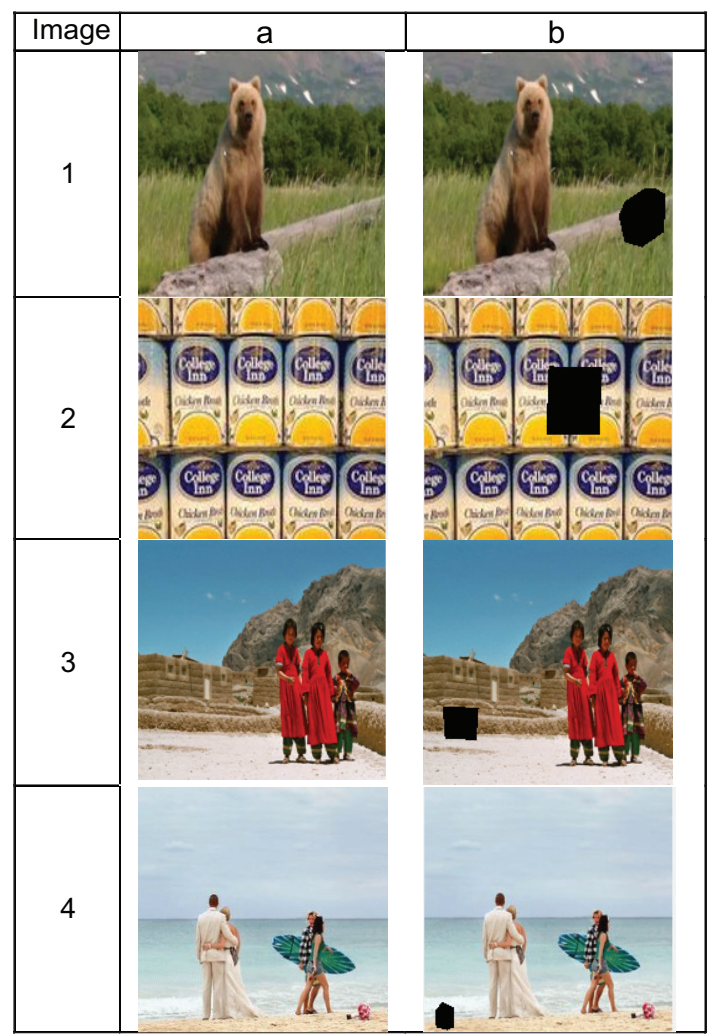

Fig. 7. Example of tested images, (a) original images, (b) original images with target region to be inpainted.

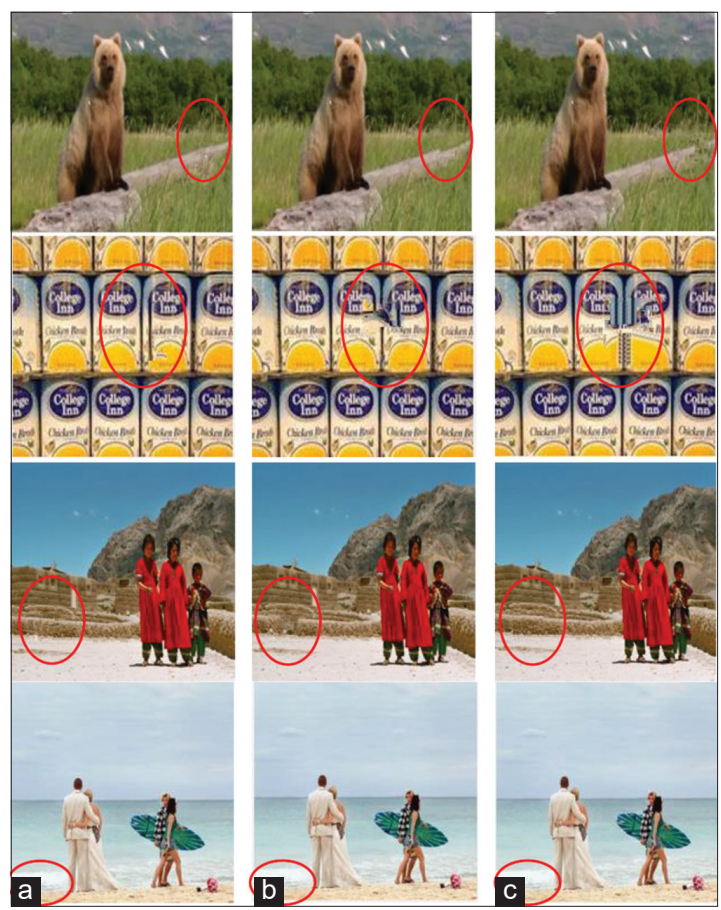

Fig. 8. Results of (a) proposed approach, (b) approach in Perez et al. [14], (c) approach in Huang et al. [18]. 
TABLE 1: Peak signal-to-noise ratio values

\begin{tabular}{lccc}
\hline Tested images & Proposed & [14] & [18] \\
\hline Image 1 & 36.5061 & 35.8802 & 36.0454 \\
Image 2 & 27.6487 & 25.4769 & 23.7248 \\
Image 3 & 35.4665 & 34.8515 & 34.0345 \\
Image 4 & 47.2478 & 45.0843 & 46.7304 \\
\hline
\end{tabular}
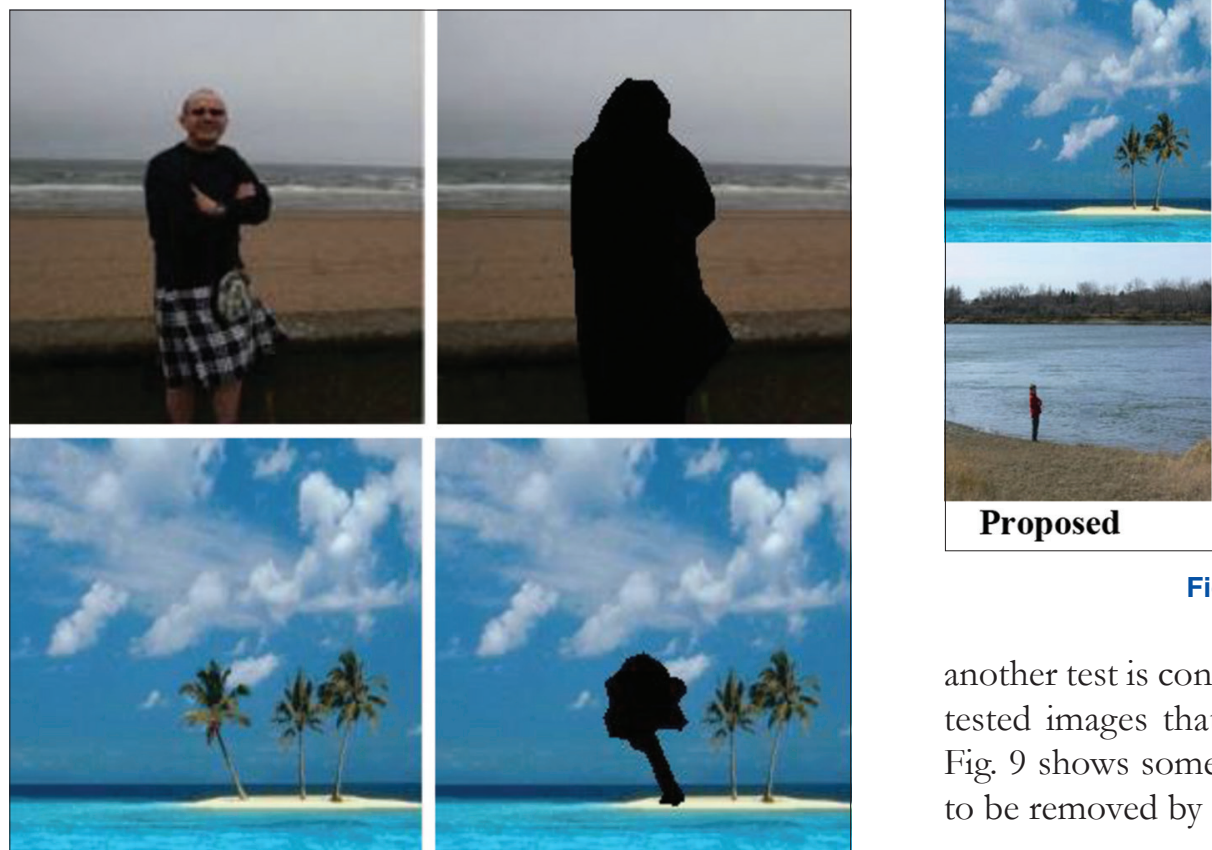

Proposed

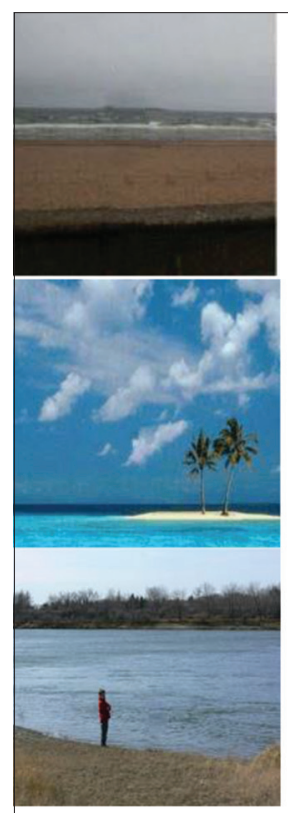

Fig. 10. Reconstructed images.

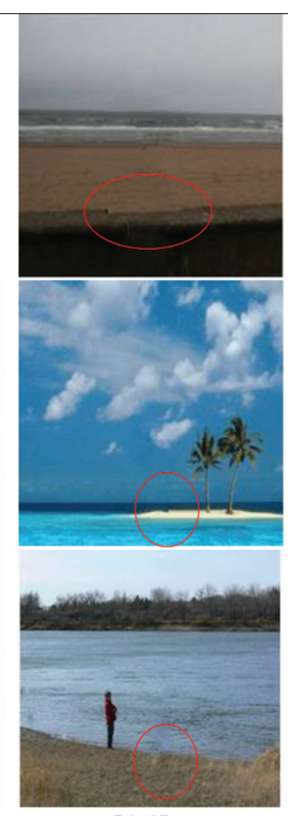

[14]

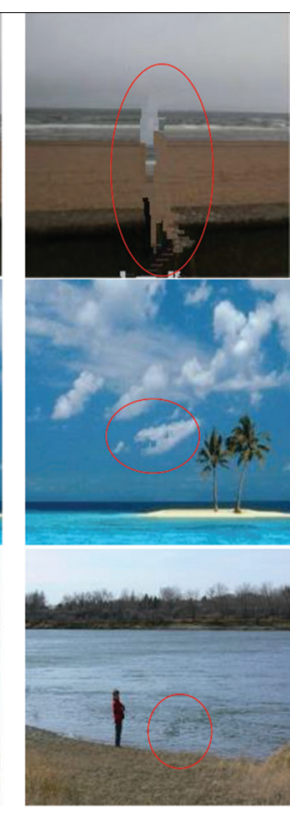

[18]

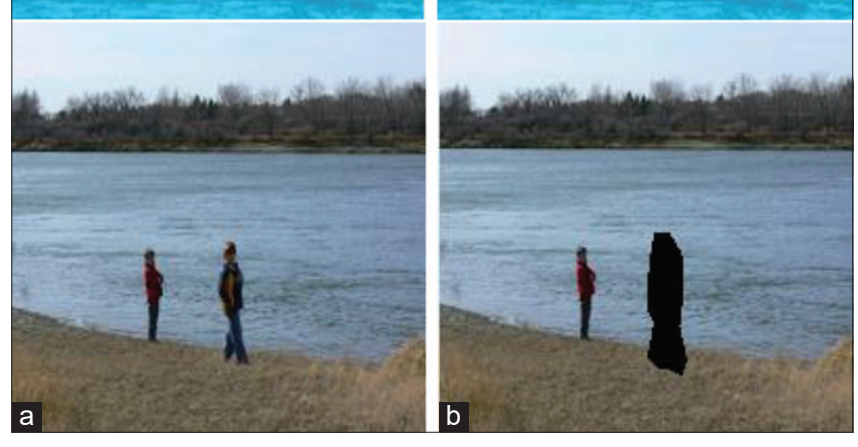

Fig. 9. (a) Original images (b) selecting unwanted objects.

Based on the results present in Table 1, one can see that sometimes the result of Perez et al. [14] is better than the result of Huang et al. [18] and vice versa, while for the proposed approach, for all tested images, the results are better than both Perez et al. [14] and Huang et al. [18]. This is because each of the tested algorithms has limitations for filling different regions such as shapes, edges, textures, and different backgrounds, while the proposed approach exceeds this limitation.

The previous results show how to fill the missing area and how to evaluate the result objectively using PSNR. Now another test is conducted by removing an object from some tested images that are evaluated subjectively, i.e., visually. Fig. 9 shows some images with selecting unwanted objects to be removed by black color.

Fig. 10 presents the results of the reconstructed images in Fig. 9(b) after filling the region of the removed objects that are marked by black color. The limitation of the reconstructed images using approaches in Perez et al. [14] and Huang et al. [18] is highlighted in a red circle. Fortunately, the proposed approach does not contain such limitation, Fig. 10.

\section{CONCLUSION}

This paper is concerned with developing a new exemplarbased image inpainting algorithm by improving the searching mechanism to find the best similar patch. The proposed algorithm is performed two steps of distances to select the best-matched patch. The first step finds the distance between the position of the selected patches and the patch which needs to be filled. On the other hand, the second step calculates the Euclidean distance between them. Finally, the summation of these two distances is calculated to find the most similar patch. Experimental results demonstrate that the proposed approach is achieved a higher quality in terms of both objectives (i.e. PSNR) and subjective (i.e., visually) compared to other existing approaches. 


\section{REFERENCES}

[1] H. Sellahewa, S. A. Jassim and A. A. Abdulla. "Stego Quality Enhancement by Message Size Reduction and Fibonacci Bitplane Mapping". United Kingdom, London, pp. 151-166, 2014.

[2] A. A. Abdulla. "Exploiting Similarities between Secret and Cover Images for Improved Embedding Efficiency and Security in Digital Steganography," Department of Applied Computing, University of Buckingham, PhD Thesis, 2015.

[3] C.Guillemot and O. Meur. "Image Inpainting: Overview and Recent advances". IEEE Signal Processing Magazine, vol. 31, no. 1, pp. 127-144, 2014.

[4] L. Cai and T.Kim. "Context-driven hybrid image inpainting". IET Image Processing, vol. 9, no. 10, pp. 866-873, 2015.

[5] B. Nizar, H. A. Ben and M. Ali. "Automatic inpainting scheme for video text detection and removal. IEEE Transactions on Image Processing, vol. 22, pp. 4460-4472, 2013.

[6] J. K. Chhabra and V. Birchha. "An enhanced technique for exemplar based image inpainting". International Journal of Computer Applications, vol. 115, pp. 20-25, 2015.

[7] R. H. Park and Y. Seunghwan. Red-eye detection and correction using inpainting in digital photographs". IEEE Transactions on Consumer Electronics, vol. 55, pp. 1006-1014, 2009.

[8] M. S. Kankanhalli and W. Q. Yan. "Erasing Video Logos Based on Image Inpainting”. Vol. 2. IEEE, Lausanne, Switzerland, pp. 521524, 2002.

[9] Wu, Y., K. Zhonglin and Z. Hongying. "An Efficient Scratches Detection and Inpainting Algorithm for old Film Restoration". Vol. 1. IEEE, Kiev, Ukraine, pp. 75-78, 2009.

[10] Y. Mecky, G. Sergios, Y. Bin and A. Karim. "Adversarial Inpainting of Medical Image Modalities". IEEE, Brighton, United Kingdom, pp. 3267-3271, 2019.

[11] M. B. Vaidya and K. Mahajan. "Image in painting techniques: A survey". IOSR Journal of Computer Engineering, vol. 5, no. 4, pp. 45-49, 2012.

[12] Jain, L., A. G. Patel and K. R. Pate. "Image inpainting-a review of the underlying different algorithms and comparative study of the inpainting techniques". International Journal of Computer Applications, vol. 118, no. 10, 2015. Available from: http://www. citeseerx.ist.psu.edu/viewdoc/download?doi=10.1.1.695.9341\&re $\mathrm{p}=$ rep1\&type $=$ pdf.

[13] B. Limbasiya and N. Pandya. "A survey on image inpainting techniques". International Journal of Current Engineering and Technology, vol. 3, no. 5, pp. 1828-1831, 2013.

[14] P. Perez, K. Toyama and A. Criminisi. "Object Removal by Exemplar-based Inpainting”. IEEE, Madison, WI, USA, 2003.

[15] P. Perez, K. Toyama and A. Criminisi. "Region filling and object removal by exemplar-based image inpainting". IEEE Transactions of Image Processing, vol. 13, no. 9, pp. 1200-12012, 2004.

[16] C. W. Hsieh, S. K. Lin, C. W. Wang and J. L. Wu, W. H. Cheng. "Robust Algorithm for Exemplar-based Image Inpainting". Proceedings of International Conference Computer Graphics, Imaging and Vision, pp. 64-69, 2005.

[17] A. Wong and J. Orchard. "A Nonlocal-means Approach to Exemplar-based Inpainting”. IEEE, San Diego, CA, USA, pp. 26002603, 2008.

[18] T. Huang, X. Zhao and L. Deng. "Exemplar-based image inpainting using a modified priority definition". Neurocomputing, vol. 10, no. 10, pp. 1-18, 2015.

[19] H. Liu, G. Lu, X. Wang, J. Wei, Y. Chao and X. Bi. "Exemplarbased Inpainting under Boundary Contraction Constraints". IEEE, Shenyang, China, pp. 295-300, 2018.

[20] A. Awati, B. Pandurngi, M. R. Patil and H. C. Rao. "Image Inpainting using Exemplar Based Technique with Improvised Data Term". IEEE, Belgaum, India, pp. 162-166, 2018.

[21] S. Wang and Y. Xu. "Image Inpainting Based on Color Differences and Structure Differences". IEEE, Dalian, China, pp. 364-368, 2013.

[22] Available from: http://www.escience.cn/people/dengliangjian/Data. html. [Last accessed on 2019 Dec 12]. 